\title{
Liquid state nuclear magnetic resonance at low fields using a nitrogen cooled superconducting quantum interference device
}

Yi Zhang, Longqing Qiu, Hans-Joachim Krause, Stefan Hartwig, Martin Burghoff, and Lutz Trahms

Citation: Appl. Phys. Lett. 90, 182503 (2007);

View online: https://doi.org/10.1063/1.2734896

View Table of Contents: http://aip.scitation.org/toc/apl/90/18

Published by the American Institute of Physics

\section{Articles you may be interested in}

Longitudinal relaxation time detection using a high- $T_{C}$ superconductive quantum interference device magnetometer

Journal of Applied Physics 102, 033914 (2007); 10.1063/1.2767193

Nuclear magnetic resonance in the earth's magnetic field using a nitrogen-cooled superconducting quantum interference device

Applied Physics Letters 91, 072505 (2007); 10.1063/1.2771060

Enhancement of nuclear magnetic resonance in microtesla magnetic field with prepolarization field detected with high- $T_{C}$ superconducting quantum interference device

Applied Physics Letters 88, 252505 (2006); 10.1063/1.2216904

Planar coil system consisting of three coil pairs for producing a uniform magnetic field Journal of Applied Physics 99, 08D904 (2006); 10.1063/1.2165107

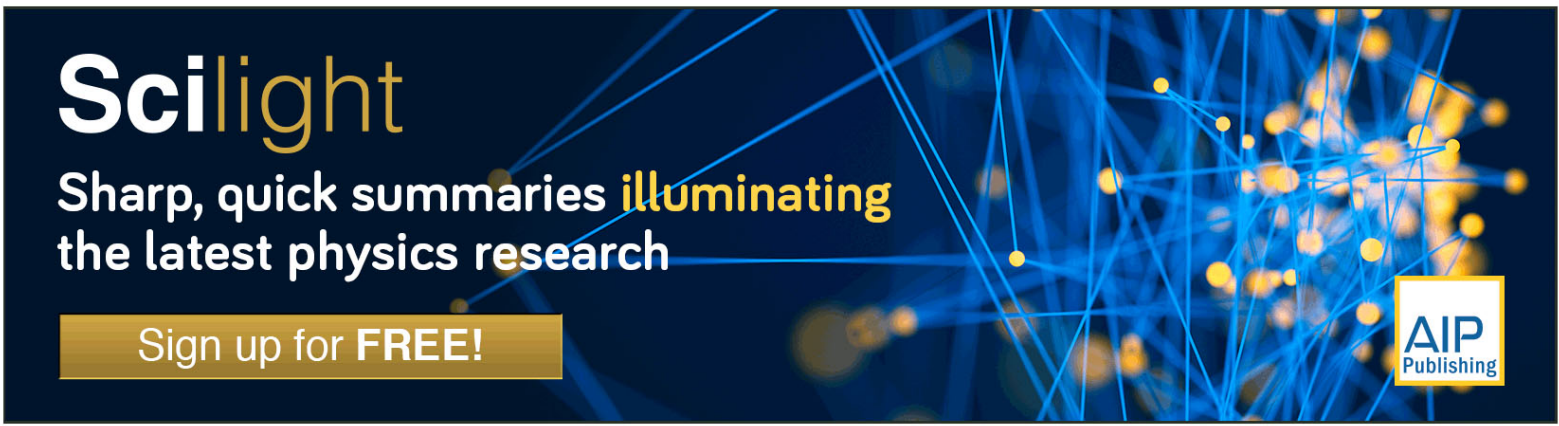




\title{
Liquid state nuclear magnetic resonance at low fields using a nitrogen cooled superconducting quantum interference device
}

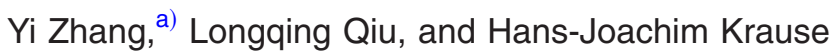 \\ Institute for Bio- and Nanosystem, Research Center Juelich, D-52425 Juelich, Germany
}

Stefan Hartwig, Martin Burghoff, and Lutz Trahms

Physikalisch-Technische Bundesanstalt (PTB), D-10587 Berlin, Germany

(Received 11 February 2007; accepted 4 April 2007; published online 1 May 2007)

\begin{abstract}
Nuclear magnetic resonance (NMR) spectra of liquids were studied at fields from $470 \mathrm{nT}$ to $65 \mu \mathrm{T}$ using a nitrogen cooled radio frequency superconducting quantum interference device. The authors demonstrated that low field NMR measurements with this device are feasible and may yield useful information. In particular, they determined the natural linewidth of distilled water to be $0.17 \pm 0.06 \mathrm{~Hz}$. In addition, they recorded $J$-coupled spectra of 2,2,2-trifluoroethanol in a measurement field regime that was determined to provide the best signal-to-noise ratio. Four peaks with frequency differences of about $2 \mathrm{~Hz}$ were well separated. (C) 2007 American Institute of Physics. [DOI: 10.1063/1.2734896]
\end{abstract}

Compared to conventional nuclear magnetic resonance (NMR) at fields of several tesla flux density, low field (LF) NMR at a few microtesla or less has the advantage of reduced requirements on the field homogeneity. For a fixed relative homogeneity, the NMR linewidth scales linearly with the measurement field and makes the NMR lines very narrow at low fields, coming close to the lifetime-limited natural linewidth. ${ }^{1}$ Furthermore, under low field conditions, chemical shifts of a few hundred ppm usually are much smaller than the lifetime-limited natural widths of the resonance lines. As a result, chemical shift information is absent in such low fields, and so is the homonuclear $J$ coupling between the degenerate states of identical nuclei. This opens the window for heteronuclear "pure $J$ spectroscopy," i.e., the investigation of electron-mediated scalar coupling between different nuclei. ${ }^{2}$

Recent studies on LF NMR utilize either a low transition temperature superconducting quantum interference device ${ }^{1-5}$ (LTS SQUID) or a high transition temperature SQUID (HTS SQUID) ${ }^{6-8}$ Recently, Yang et al. reported NMR measurements using HTS SQUIDs with a minimum measuring magnetic field of about $9 \mu \mathrm{T}$, corresponding to a proton resonance frequency of $380 \mathrm{~Hz} .{ }^{9}$ Here, we report on NMR in fields down to about $500 \mathrm{nT}$ flux density, using a HTS radio frequency (rf) SQUID. In particular, we address the problem of how to deal with the higher noise of HTS SQUIDs, with the aim to obtain the best possible results in terms of well resolved spectra with a reasonable signal-to-noise ratio (SNR).

The rf SQUID magnetometer used for this work is a so-called substrate resonator version. ${ }^{10} \mathrm{~A}$ yttrium barium copper oxide thin film SQUID washer structure is patterned on the $\mathrm{SrTiO}_{3}$ substrate with the dimensions of $10 \times 10$ $\times 1 \mathrm{~mm}^{3}$, which serves as a tank circuit (resonator) and as a flux concentrator. On the resonator substrate, a small rf washer SQUID with a loop of $150 \times 150 \mu \mathrm{m}^{2}$ is positioned in flip-chip geometry. This SQUID magnetometer achieved a field-to-flux transfer coefficient $\partial B / \partial \phi$ of $1.85 \mathrm{nT} / \Phi_{0}$. White

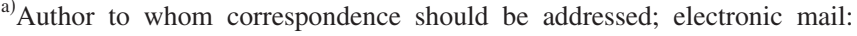
y.zhang@fz-juelich.de
SQUID flux noise of $20 \mu \Phi_{0} / \sqrt{ } \mathrm{Hz}$ in this work corresponded to a field sensitivity of about $40 \mathrm{fT} / \sqrt{ } \mathrm{Hz}$ down to about $10 \mathrm{~Hz}$. The magnetometer was operated in flux locked loop mode. The SQUID was mounted at the bottom of a Dewar vessel filled with liquid nitrogen.

The measurements were performed in the Berlin magnetically shielded room, which has a residual field below $10 \mathrm{nT} .^{11}$ Instrumental setup and experimental procedure are sketched in Fig. 1. The liquid sample at room temperature was located beneath the SQUID magnetometer and inserted into a polarizing coil. The coil set consists of three solenoids: a polarizing coil with the sample in the center is complemented by two small solenoids at the sides, connected in series with opposite polarity. These two small solenoids compensate the magnetic field at larger distances in order to avoid the magnetization of the walls of the magnetically shielding room. The SQUID, the polarizing coil set, and the sample were positioned in the center of a Helmholtz coil with a diameter of $1 \mathrm{~m}$ which is used to generate the detection field $B_{m}$ along the $y$ direction, perpendicular to the polarizing field $B_{p}$. To avoid flux trapping into the SQUID, both $B_{p}$ and $B_{m}$ are parallel to the plane of the SQUID. The vertical component of the magnetic field that is generated by the precessing nuclear magnetization was detected by the SQUID at its location some $10 \mathrm{~mm}$ above the upper level of the liquid in the sample tube (see Fig. 1 left).

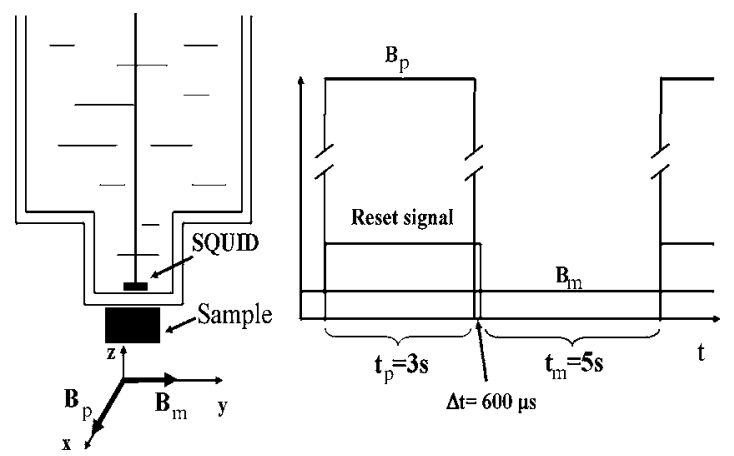

FIG. 1. Arrangement and pulse sequence of the experiment. 

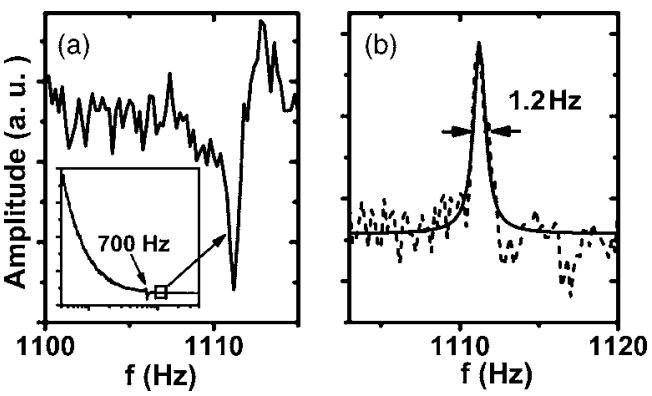

FIG. 2. Typical process of signal acquisition. (a) Fourier spectrum of measured NMR signal for $20 \mathrm{ml}$ distilled water after averaging 100 times, in the frequency range around its Larmor frequency. The inset shows the full spectrum. (b) Phase corrected spectrum and fit of a Lorentzian curve to the data.

The measurement was started by polarizing the nuclear magnetization of the sample in a field of about $B_{p}=1 \mathrm{mT}$, which was generated by the polarizing coil for $3 \mathrm{~s}$, a time which is longer than the longitudinal relaxation time constant $\left(T_{1}\right)$ of the water. ${ }^{12}$ After $B_{p}$ was switched off, the sample was left in the much weaker $B_{m}$ which was continuously applied during the experiment. During the polarizing time of $3 \mathrm{~s}$ and during the first $600 \mu \mathrm{s}$ after switch off of the polarizing field coil, the SQUID readout electronics was kept in the reset state, in which the SQUID was kept in an open loop while the polarizing field was still high. Subsequently, the SQUID was locked in order to record the $z$ component of the magnetic field generated by the precession of the nuclear magnetization NMR signal.

We measured the NMR signal of $20 \mathrm{ml}$ distilled water. The measurement field $B_{m}$ was varied from $470 \mathrm{nT}$ to $65 \mu \mathrm{T}$, or, in terms of the proton Larmor frequency $f_{L}$, between $20 \mathrm{~Hz}$ and $2.8 \mathrm{kHz}$. Figure 2(a) shows the NMR spectrum at $B_{m}=26 \mu \mathrm{T}$, around the Larmor frequency $f_{L}$ of about $1.11 \mathrm{kHz}$. It was transformed from the average of 100 relaxation decays. Towards lower frequencies the spectral base line increases (see insert), probably due to the magnetization decay of the walls of the magnetically shielded room after the polarizing field $B_{p}$, is switched off. This perturbation was much smaller in our earlier low field NMR study of water, where we could suppress the interference by gradiometer compensation. ${ }^{3}$ The origin of the big peak near $700 \mathrm{~Hz}$ should be attributed to a resonance in the coil system. After a phase correction the NMR line can be fitted by a Lorentzian peak having a linewidth of $1.2 \mathrm{~Hz}$, as shown in Fig. 2(b).

The dependence of the linewidth $\Delta \nu$ on $f_{L}$ is shown in Fig. 3. We can see that $\Delta \nu$ increases linearly with $\Delta \nu\left(f_{L}\right)$ $=0.000924 f_{L}+0.166 \mathrm{~Hz}$. Below $100 \mathrm{~Hz}$, the increasing $1 / f$ noise of the recording compromises the accuracy of the determination of $\Delta \nu$. The slope of $\alpha=924 \pm 55 \mathrm{ppm}$ is about two times higher than in our earlier LTS SQUID NMR study. ${ }^{3}$ This is not surprising because the slopes are instrumental parameters, which reflect the different inhomogeneities of the two setups. On the other hand, the constant contribution of $\beta=166 \pm 59 \mathrm{mHz}$, which is given by the intersection of the regression line with the $f_{L}=0$ axis in Fig. 3 , is in good agreement with the value we found for distilled water with the LTS SQUID NMR setup having much less noise. ${ }^{3}$ This is also expected, because $\beta$ reflects the natural linewidth of the investigated liquid in the absence of instrumental broadening, and should be independent of the measurement device. Nevertheless, we find it remarkable that

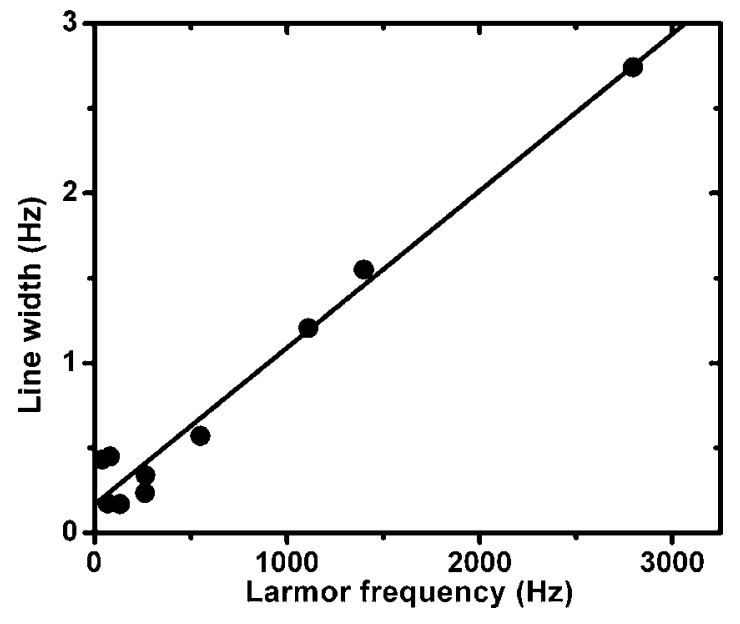

FIG. 3. Relationship between ${ }^{1} \mathrm{H}$ Lorentzian linewidths and their Larmor frequencies.

one can determine natural NMR linewidths of liquids in the range of $0.1 \mathrm{~Hz}$ with a reasonable accuracy using HTS SQUID spectrometers having a much higher noise level than LTS devices.

The natural linewidth reflects the lifetime of the transversal magnetization, $T_{2}=1 / \pi \Delta \nu$. It was recognized already by the pioneers of magnetic resonance that nuclear magnetization relaxes due to magnetic field fluctuations in resonance with the Larmor frequency. ${ }^{13}$ By this mechanism, relaxometry provides insight into the spectral distribution of intraand intermolecular motions in liquids. The low frequency range of a few kilohertz or less can be covered by the socalled field-cycling technique, where the field strength is switched rapidly between several teslas and a few milli- or microteslas. ${ }^{14,15}$ In the past, most of these investigations focused on the longitudinal relaxation time $T_{1}$, while the measurement of $T_{2}$ remained difficult even for the elaborate field-cycling technology. Now, LTS and possibly even HTS SQUID-based low field NMR offers a new and, in some respect, much easier access to $T_{2}$ at low frequencies (and to $T_{1}$ as well).

It was pointed out by McDermott et al. ${ }^{1}$ that the reduced linewidth at lower field strengths gives rise to an improved SNR. For the magnetization that is generated by the constant polarization field of $1 \mathrm{mT}$, the integral intensity of the peak remains unchanged. Thus, the linear increase of the linewidth causes a decrease of the amplitude with the detection field. Accordingly, the SNR of our NMR spectra should increase towards lower detection fields. This improvement at lower Larmor frequencies, however, is compromised by the $1 / f$ noise of SQUIDs, which also increases towards lower frequencies (see Fig. 4). As a result, there exists a window of the detection field for every SQUID-based low field NMR spectrometer where the best SNR can be achieved. In our case, spectra with a reasonable SNR can be obtained between 3 and $10 \mu \mathrm{T}$, i.e., between proton Larmor frequencies of 120 and $400 \mathrm{~Hz}$.

In Fig. 5, we present a spectrum of $20 \mathrm{ml} \mathrm{2,2,2-}$ trifluoroethanol $\left(\mathrm{CF}_{3}-\mathrm{CH}_{2}-\mathrm{OH}\right)$ obtained by Fourier transformation of 400 averaged magnetization decays taken at $3.13 \mu \mathrm{T}$. As discussed in detail elsewhere, ${ }^{2-4}$ the rightmost peak at about $138 \mathrm{~Hz}$ belongs to the proton of the $\mathrm{OH}$ group and precesses with its unperturbed Larmor frequency, because it is in fast exchange and does not couple to the fluo- 


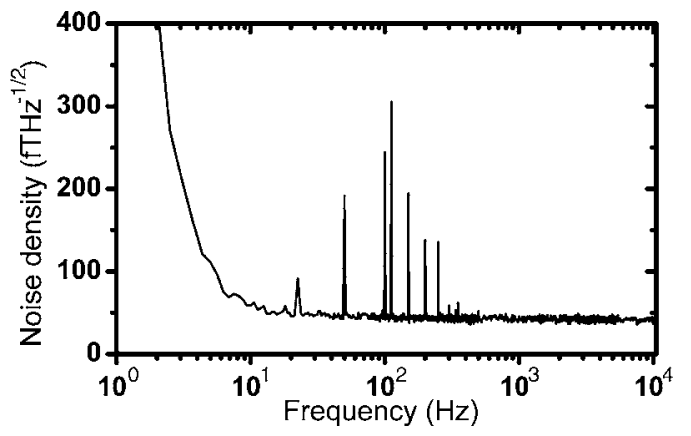

FIG. 4. Power spectrum of the HTS SQUID from $1 \mathrm{~Hz}$ to $10 \mathrm{kHz}$.

rine nuclei. The other peaks at 130,133 , and $136 \mathrm{~Hz}$ reflect $J$-coupled states of fluorine and proton spins which cannot be assigned to individual nuclei. Natural lifetime broadening of about $0.4 \mathrm{~Hz}$ conceals that in reality these three peaks are superpositions of many more states indicated by the stick spectra shown in the bottom line of Fig. 5 caused by heteronuclear $J$ couplings ${ }^{16}$ with $J=8.9 \mathrm{~Hz}$ for the ${ }^{19} \mathrm{~F}-{ }^{1} \mathrm{H}$ system, in trifluoroethanol. ${ }^{3}$

In conclusion, we have shown that the proportionality between instrumental broadening and Larmor frequency can be utilized for the determination of the natural linewidth by extrapolation to zero field, where instrumental broadening vanishes. In addition, we demonstrated that the trade off between the increase of the $1 / f$ noise towards lower frequencies and the decrease of the peak amplitude at higher Larmor frequencies defines the best field and Larmor frequency range for SQUID-based low field NMR spectroscopy. The broadband detection characteristics of SQUIDs gives the freedom to choose the appropriate Larmor frequency in a wide range. By making use of this benefit, we have recorded NMR spectra at low fields with HTS rf SQUID operated at $77 \mathrm{~K}$ with results comparable to data obtained by liquidhelium cooled LTS SQUIDs. ${ }^{2}$ Such spectra may be used as

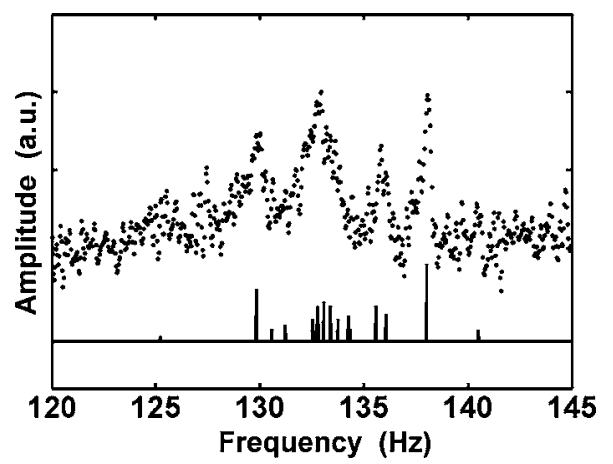

FIG. 5. $J$-coupling spectrum of 2,2,2-trifluoroethanol at a $B_{m}$ of $3.13 \mu \mathrm{T}$. The stick spectra in the bottom line are simulated using a coupling constant $J=8.9 \mathrm{~Hz}$. fingerprints for identifying particular substances in liquids. One may object that this application is limited by the poor sensitivity of low field NMR, but we find it encouraging that we were able here to resolve the trifluoroethanol spectrum even with a HTS SQUID.

There are still two means left for improving the signalto-noise ratio of SQUID-based low field NMR relaxometry and spectroscopy: the increase of the filling factor and the increase of the polarizing field. We employed a mu-metal shielded room to provide the condition for the generation of low fields around a microtesla, where favorable conditions for both pure $J$ spectroscopy and high resolution $T_{2}$ relaxometry prevail. In principle, the Earth's field and other magnetic perturbations can also be reduced by feedback controlled compensation coils. This may make low field NMR not only less expensive but also more effective, because in this situation one does not have to take heed of the magnetization of mu-metal walls by the polarizing field. Since the nuclear magnetization scales linearly with the polarizing field, this can improve the signal-to-noise ratio considerably; with our HTS-SQUID device operating outdoors we measured the nuclear precession of $20 \mathrm{ml}$ water in the Earth's magnetic field after polarization in a field of $10 \mathrm{mT}$ and obtained a SNR of 20 in a single shot.

${ }^{1}$ R. McDermott, A. H. Trabesinger, M. Mück, E. L. Hahn, A. Pines, and J. Clarke, Science 295, 2247 (2002).

${ }^{2}$ J. Bernarding, G. Buntkowsky, S. Macholl, S. Hartwig, M. Burghoff, and L. Trahms, J. Am. Chem. Soc. 128, 714 (2006).

${ }^{3}$ M. Burghoff, S. Hartwig, L. Trahms, and J. Bernarding, Appl. Phys. Lett. 87, 054103 (2005).

${ }^{4}$ A. H. Trabesinger, R. McDermott, S. Lee, M. Mück, J. Clarke, and A. Pines, J. Phys. Chem. A 108, 957 (2004).

${ }^{5}$ J. J. Heckman, M. P. Ledbetter, and M. V. Romalis, Phys. Rev. Lett. 91, 067601 (2003)

${ }^{6}$ K. Schlenga, R. F. McDermott, J. Clarke, R. E. de Souza, A. Wong-Foy, and A. Pines, IEEE Trans. Appl. Supercond. 9, 4424 (1999).

${ }^{7}$ S. Kumar, R. Matthews, S. G. Haupt, D. K. Lathrop, M. Takigawa, J. R. Rozen, S. L. Brown, and R. H. Koch, Appl. Phys. Lett. 70, 1037 (1997).

${ }^{8}$ K. Schlenga, R. McDermott, John Clarke, R. E. de Souza, A. Wong-Foy, and A. Pines, Appl. Phys. Lett. 75, 3695 (1999).

${ }^{9}$ H.-C. Yang, S.-H. Liao, H.-E. Horng, S.-L. Kuo, H.-H. Chen, and S. Y. Yang, Appl. Phys. Lett. 88, 252505 (2006).

${ }^{10}$ Y. Zhang, N. Wolters, J. Schubert, D. Lomparski, M. Banzet, G. Panaitov, H.-J. Krause, M. Mück, and A. I. Braginski, IEEE Trans. Appl. Supercond. 13, 389 (2003).

${ }^{11}$ S. N. Erne, H. D. Hahlbohm, J. Scheer, and Z. Trontelj, in Biomagnetism, edited by S. N. Erne, H. D. Hahlbohm, and H. Lübbig (deGruyter, Berlin, 1981), pp. 79-87.

${ }^{12}$ V. Graf and F. Noack, J. Chem. Phys. 72, 861 (1980).

${ }^{13}$ N. Bloembergen, E. M. Purcell, and R. V. Pound, Phys. Rev. 73, 679 (1948).

${ }^{14}$ R. Kimmich and E. Anoardo, Prog. Nucl. Magn. Reson. Spectrosc. 44, 257 (2004).

${ }^{15}$ F. Noack, Prog. Nucl. Magn. Reson. Spectrosc. 18, 171 (1986).

${ }^{16} \mathrm{P}$. L. Corio, Structure of High-Resolution NMR Spectra (Academic, New York, 1966). 